\title{
Qualidade das informações de saúde e medicamentos nos sítios brasileiros
}

\author{
Quality of health and medication information on Brazilian websites
}

\author{
Ana Paula Soares Gondim ${ }^{1}$, Davi Pontes Weyne ${ }^{1}$, Bruno Sousa Pinto Ferreira ${ }^{1}$
}

\section{RESUMO}

Objetivo: Avaliar a qualidade das informações sobre saúde e medicamentos disponíveis em sítios brasileiros. Métodos: Estudo descritivo, de abordagem quantitativa em sítios brasileiros, realizado no período de janeiro a março de 2011. A localização dos sítios de busca ocorreu empregando duas palavras-chave: "informações sobre medicamentos" e "informações sobre saúde". A escolha das variáveis baseou-se nos critérios da qualidade da informação na internet da Organização Mundial da Saúde e do Código Internacional de Ética para sítios de saúde e serviços na internet. A variável dependente foi 0 sítio que apresentava informação sobre medicamento ou sobre saúde. As variáveis independentes foram acesso, aparência, organização, honestidade, transparência, responsabilidade e procedência. Para análise estatística, aplicaram-se os testes do $\chi^{2}$ e exato de Fisher, com nível de significância de $5 \%$. Resultados: Dos 37 sítios brasileiros analisados, $24(64,9 \%)$ eram de informações para saúde e $13(35,1 \%)$ sobre medicamentos. Nos critérios de acesso, aparência e organização, a maioria dos sítios sobre saúde e sobre medicamentos era de fácil acesso, fácil entendimento, linguagem objetiva, atualizados e organizados de forma lógica e fornecia informação exata e cientificamente fundamentada. Conclusão: 0 critério honestidade apresentou diferença estatisticamente significante entre os sítios. A qualidade das informações geradas nos sítios sobre saúde e sobre medicamentos apresentou diferenças importantes, exigindo uma organização mais sistemática desses temas apresentados em seus ciberespaços.

Descritores: Internet; Informação de saúde ao consumidor; Produtos e serviços de informação

\section{ABSTRACT}

Objective: To evaluate the quality of information about health and medication available on Brazilian websites. Methods: A descriptive study with a quantitative approach regarding Brazilian websites, conducted from January to March 2011. The search sites were located using two search phrases: "medication information" and "health information." The choice of variables was based on the Internet information quality criteria of the World Health Organization and the International Code of Ethics for health and services sites on the Internet. The dependent variable was whether the site had information about health or medication. The independent variables were access, appearance, organization, honesty, transparency, responsibility and origin. For statistical analysis, the $\chi^{2}$ and Fisher exact tests were applied, with a significance level of $5 \%$. Results: Of the 37 Brazilian sites analyzed, $24(64.9 \%)$ contained health information and $13(35.1 \%)$ contained medication information. Regarding appearance, organization and access criteria, most sites related to health and medication were easily accessible, easy to understand, used objective language, were updated and organized logically and provided accurate and scientifically grounded information. Conclusion: The honesty criterion differed significantly between sites, and the quality of information presented on health and medication websites showed significant differences, suggesting the need for a more systematic organization of these topics on the Internet.

Keywords: Internet; Consumer health information; Information products and services

\section{INTRODUÇÃo}

Nas últimas duas décadas, verificou-se o crescimento exponencial de pessoas que usam informações pela internet, atingindo todas as áreas, particularmente a saúde. A prevalência de sítios de buscas na internet por problemas de saúde é estimada em 4,5\% entre todas as buscas que podem estar relacionada à saúde ${ }^{(1,2)}$.

Nos Estados Unidos, duas pesquisas revelaram que mais de $63 \%$ dos usuários usam, pelo menos uma vez

\footnotetext{
Trabalho realizado na Universidade de Fortaleza - UNIFOR, Fortaleza (CE), Brasil.

1 Universidade de Fortaleza - UNIFOR, Fortaleza (CE), Brasil.

Autor correspondente: Ana Paula Soares Gondim - Avenida Washington Soares, 1.321 - Edson Queiroz - CEP: $60811-905$ - Fortaleza (CE), Brasil - Tel.: (85) 3477-3280 - E-mail: anapaulasgondim@ @unifor.br Data de submissão: 27/11/2011 - Data de aceite: 28/2/2012

Conflitos de interesse: não há.

Fonte de financiamento: bolsa de iniciação científica do Conselho Nacional de Pesquisa e Desenvolvimento Tecnológico (CNPq).
} 
por ano, a internet para obter informações sobre saú$\mathrm{de}^{(3,4)}$. Os motivos são a possibilidade direta da melhoria da saúde ou a contestação da base de dados médicos, confrontando-os com "histórias" da internet. Estudos realizados nos Estados Unidos e na Europa apontam que uma pequena proporção de médicos ou profissionais de saúde use sítios de busca comercial para procurar assuntos relacionados à saúde $\mathrm{e}^{(5)}$.

No Brasil, estudo mostrou que as farmácias virtuais brasileiras facilitam o acesso a medicamentos não registrados e o descontrole sobre a venda de medicamentos de controle especial, tornando-se possível o uso inadequado e indiscriminado de medicamentos, apontando para uma insuficiência de recursos humanos e falha de regulamentação da Agência Nacional de Vigilância Sanitária (ANVISA). Também, verificou-se que a principal fonte de informação presente nos sítios eram as bulas de medicamentos ${ }^{(6)}$.

Os riscos das informações de saúde e medicamentos na internet apontam para mudanças na relação médico-paciente nas ações do paciente frente à sua enfermidade: mais de $70 \%$ dos usuários afirmam que as informações contidas na internet influenciam suas decisões sobre o tratamento prescrito $^{(7)}$. Além disso, o mercado farmacêutico reforça o uso da informação pela internet, por meio da promoção de estratégias de credibilidade para seus produtos e, consequentemente, maior consumo, que pode levar a efeitos adversos ou intoxicações por medicamentos ${ }^{(8)}$.

Nos Estados Unidos, estima-se um crescimento farmacêutico de 800 bilhões de dólares em 2010, podendo ser ainda maior até $2013^{(9)}$, enquanto no Brasil, estima-se um crescimento de 15,4 bilhões de dólares ${ }^{(10)}$. Essa constatação evidencia que é necessário avaliar permanentemente a qualidade da informação de saúde na internet. Indiscutivelmente, os aspectos éticos são indispensáveis para boa prestação de cuidados de saúde de qualidade, sendo a produção de informação pela internet um desafio para a clínica ${ }^{(11)}$. Aprovou-se, no senado americano, em 2000, o Código Internacional de Ética para sítios e serviços de saúde na internet ${ }^{(12)}$, também adotado na Europa e em outros países, embora sejam recomendadas normas voluntárias, não vinculadas a uma organização e que norteiem atividades éticas e sociais ${ }^{(13)}$.

Na área da saúde, a preocupação com a qualidade é constante. Desde 1997, a Agency for Health Care Policy and Research, juntamente do Health Information Technology Institute (HITI), e a empresa Mitretek Systems por intermédio do Health Summit Working Group, elaboraram um documento, intitulado Criteria for the Quality of Health Information on the Internet, que apre- senta sete critérios para se avaliar a qualidade da informação em sítios de informações sobre saúde: credibilidade, conteúdo, apresentação do sítio, links, design, interatividade e anúncios. Tem, ainda, como propósito, subsidiar o consumidor da rede na escolha e na identificação de sítios confiáveis ou com fraudes ${ }^{(14)}$.

Uma das instituições mais conhecidas, a Internet Healthcare Coalition, que defende a promoção de recursos de saúde de qualidade na internet, é representada por consumidores americanos, defensores de pacientes, criadores comerciais de informação em saúde, profissionais de saúde, bibliotecários médicos, funcionários do governo e fabricantes de produtos farmacêuticos. Tem como objetivo educar consumidores, profissionais de saúde e outros sobre as questões relativas à evolução da qualidade dos recursos de internet de saúde e de informação ${ }^{(15)}$.

A Organização Mundial da Saúde (OMS) determina que a informação disponível na internet deva estabelecer o propósito do sítio (se é educativo ou possui fins comerciais), definindo, assim, seu público-alvo ${ }^{(16)}$. A informação deve conter a data e a procedência da publicação (instituições, organizações ou mesmo os fabricantes que se responsabilizam legal e eticamente por disponibilizar as informações), esclarecer se há referências de estudos clínicos e mencionar grupos ou entidades de portadores de uma determinada doença que apoiam a publicação dessas informações.

Algumas entidades, como a Associação Médica Americana (AMA), desenvolveram diretrizes para orientar a busca de informações em sítios na internet, desde sua aquisição do sítio até a publicidade e patrocínio on-line da informação e do produto, assegurando, aos visitantes doentes, privacidade e confidencialidade. Além disso, os sítios devem fornecer meios eficazes e seguros para comercialização de produtos Essas diretrizes favorecem o próprio sítio da AMA, como outros provedores. Ocorrem atualizações frequentes nos objetos da AMA, acompanhando a evolução tecnológica e novas práticas da internet ${ }^{(17)}$.

O Conselho Federal de Medicina elaborou um documento com nove normas para assegurar a qualidade da informação da rede: integridade da informação e do serviço; privacidade; confidencialidade; autenticação; auditoria; honestidade; segurança; transparência e consentimento livre e esclarecido ${ }^{(18)}$. Destaca-se, também, o Conselho Regional de Medicina do Estado de São Paulo (CREMESP), que publicou um manual de princípios éticos para sítios de medicina e saúde, que orienta o usuário na busca de informações, serviços ou produtos de saúde on-line. Além disso, informa o direito de exigir das organizações e indivíduos responsáveis pelos 
sítios: transparência, honestidade, qualidade, consentimento livre e esclarecido, privacidade, ética médica, responsabilidade e procedência ${ }^{(19)}$.

A importância do ciberespaço como fonte e atuação da informação sobre saúde e medicamentos pode ajudar a direcionar para uma reflexão de um consenso, tanto para os profissionais de saúde como para os usuários que buscam a internet como fonte de informação.

\section{OBJETIVO}

Este artigo visa avaliar diferenças na qualidade das informações sobre saúde e medicamentos disponíveis em sítios brasileiros.

\section{MÉTODOS}

Estudo descritivo, de abordagem quantitativa em sítios brasileiros com informações sobre saúde e medicamentos. O programa Microsoft Internet Explorer ${ }^{\circledR}$, versão 6.0, foi empregado para visualizar websites por meio de seus endereços Uniform Resource Locator (URL). Selecionou-se o sítio de busca Google por ser o mais utilizado no mundo ${ }^{(20)}$.

A pesquisa dos sítios ocorreu no período de janeiro a março de 2011, sendo identificados 286 sítios de busca sobre saúde e medicamento, empregando duas palavras-chave: "informações sobre medicamentos" e "informações sobre saúde". Os sítios com "informações sobre saúde" apresentavam imagens ou textos sobre saúde, bem-estar, promoção da saúde ou doenças específicas. Os sítios com "informações sobre medicamentos" apresentavam imagens ou textos de produtos farmacêuticos ou medicamentos.

De acordo com os critérios adotados (endereços URL com extensão.br e apresentar informações sobre saúde e medicamentos direcionadas aos profissionais da saúde e usuários), foram identificados 37 sítios brasileiros. Os critérios de exclusão foram: páginas voltadas apenas às propagandas e às vendas de produtos e medicamentos; sítios de hospitais, organizações não governamentais, conselhos, associações e órgãos governamentais; sítios que traziam informações sobre medicamentos veterinários ou cosméticos; e páginas fora do ar ou que não traziam informações sobre saúde e medicamentos.

Foram analisadas sete variáveis neste estudo; com base nos critérios da qualidade da informação na internet da $\mathrm{OMS}^{(16)}$ e do Código Internacional de Ética para sítios de saúde e serviços na internet ${ }^{(12)}$, elaborou-se um formulário para coletar os dados (Anexo 1).

A variável dependente foi o sítio que apresentava informação sobre medicamento ou sobre saúde. As va- riáveis independentes foram: acesso explicado como fácil acesso, links para outros sítios relevantes com alguma especialidade e quantidade de usuários que acessam; aparência explicada por visibilidade da data de publicação ou revisão, citação das fontes utilizadas, fácil entendimento e de linguagem objetiva, exata e cientificamente fundamentada; organização explicada pela atualização do sítio e organização lógica das informações; honestidade explicada, pelo propósito do sítio, por ser verdadeiro e não enganador, por meio da interferência dos autores e patrocinadores; transparência explicada pela responsabilidade da seleção do conteúdo, que não deve por em risco a privacidade das informações dos usuários, e nem apresentar chat, espaço para dúvidas, reclamações e sugestões, algum efeito visual ou sonoro que desvie a atenção do entendimento das informações e voltado para alguma especialidade; responsabilidade explicada como responsável pela criação e atualização do sítio; presença dos patrocinadores, responsável técnico identificado, informações ilógicas da boa compreensão terapêutica e farmacocinética; artigos submetidos a um editor de revisão; procedência explicada como fontes profissionais, entidades, universidades, órgãos públicos (município, estadual e federal); órgãos privados e outras instituições reconhecidamente qualificadas.

Os dados foram inseridos em um banco de dados e analisados no programa Statistical Package for the Social Sciences 14.0 for Windows Student Version ${ }^{\circledR}$ (EUA). Consistiu de uma análise estatística com resultados expressos em proporção, tendo sido aplicados os testes do $\chi^{2}$ e exato de Fisher, com nível de significância de 5\%, para avaliar as diferenças entre os sítios de informações sobre saúde e os de informações sobre medicamentos. Adotou-se o valor sim e não nos itens que compõem cada variável.

\section{RESULTADOS}

Dos 37 sítios brasileiros analisados, $24(64,9 \%)$ foram de informações para saúde e $13(35,1 \%)$ de informações sobre medicamentos.

Nos critérios de acesso, aparência e organização, a maioria dos sítios sobre saúde e sobre medicamentos era de fácil acesso e entendimento, tinha linguagem objetiva, e informação exata e cientificamente fundamentada, e era atualizado e organizado de forma lógica. Os sítios sobre medicamentos apresentaram baixa visibilidade de publicação ou revisão $(15,4 \%)$. A maioria dos sítios sobre saúde teve elevada proporção para citação da fonte utilizada $(69,2 \%)$ (Tabela 1$)$. 
Tabela 1. Acesso, aparência e organização

\begin{tabular}{|c|c|c|c|}
\hline \multirow{2}{*}{ Variáveis } & Sítio sobre saúde $(n=24)$ & Sítio sobre medicamento $(n=13)$ & \multirow{2}{*}{ Valor de $\mathrm{p}$} \\
\hline & n (\%) & n (\%) & \\
\hline Fácil acesso & $15(62,5)$ & $10(76,9)$ & 0,476 \\
\hline Links para outros sítios relevantes & $9(37,5)$ & $6(46,2)$ & 0,730 \\
\hline Quantidade de usuários que acessaram & $3(12,5)$ & $1(7,7)$ & 1,000 \\
\hline Visibilidade da data de publicação ou revisão & $13(54,2)$ & $2(15,4)$ & 0,035 \\
\hline Citação das fontes utilizadas & $9(37,5)$ & $9(69,2)$ & 0,091 \\
\hline Fácil entendimento e linguagem objetiva & $15(62,5)$ & $9(69,2)$ & 0,734 \\
\hline Exata e cientificamente fundamentada & $12(50,0)$ & $10(76,9)$ & 0,165 \\
\hline Atualização do sítio & $17(70,8)$ & $8(61,5)$ & 0,716 \\
\hline Organizado de forma lógica & $13(54,2)$ & $7(53,8)$ & 1,000 \\
\hline
\end{tabular}

O número de acesso variava nos sítios - em alguns chegavam a 74.000 usuários por dia.

Nenhum dos critérios de acesso apresentou diferença estatisticamente significante entre os sítios investigados. Entre os critérios de aparência, visibilidade da data de publicação ou revisão $(p=0,035)$ mostrou-se estatisticamente significante entre os sítios investigados (Tabela 1).

Nos critérios de honestidade, transparência e responsabilidade, a maioria dos sítios sobre saúde e sobre medicamentos tinha o responsável pela seleção do conteúdo e não colocava em risco a privacidade dos usuários. A maioria $(62,5 \%)$ dos sítios sobre saúde não tinha chat, espaço para dúvidas, reclamações, sugestões e links para outros sítios relevantes, como voltado para alguma especialidade. A maioria dos sítios sobre medicamentos teve elevada proporção para indicação de patrocinadores $(92,3 \%)$ e interferência dos patrocina- dores $(69,2 \%)$. Verificou-se uma proporção muito baixa $(8,3 \%)$ nos sítios sobre saúde em relação ao propósito do sítio. Nos sítios sobre saúde, $25 \%$ indicavam o responsável técnico e, nos sítios sobre medicamentos, esse número foi mais elevado $(46,2 \%)$. Mais de $15 \%$ das informações, tanto nos sítios sobre saúde como nos sítios sobre medicamentos, são ilógicas para boa compreensão terapêutica e farmacocinética (Tabela 2).

Evidenciaram-se diferenças estatisticamente significantes em todos os critérios de honestidade entre os sítios: interferência dos autores das informações e dos patrocinadores no conteúdo $(\mathrm{p}<0,001)$ e linha editorial do sítio $(0,002)$. Nos critérios de transparência entre os sítios, não se observaram diferenças estatisticamente significantes. Nos critérios de responsabilidade, constataram-se diferenças estatisticamente significantes em responsabilidade pela criação do sítio $(\mathrm{p}=0,005)$ e patrocinadores do conteúdo $(\mathrm{p}<0,001)$ (Tabela 2).

Tabela 2. Critérios de honestidade, transparência e responsabilidade

\begin{tabular}{|c|c|c|c|}
\hline \multirow{2}{*}{ Variáveis } & \multirow{2}{*}{$\begin{array}{c}\text { Sítio sobre saúde }(n=24) \\
n(\%)\end{array}$} & \multirow{2}{*}{$\begin{array}{c}\text { Sítio sobre medicamento }(n=13) \\
n(\%)\end{array}$} & \multirow{2}{*}{ Valor de $\mathbf{p}$} \\
\hline & & & \\
\hline Interferência dos autores e patrocinadores & $5(20,8)$ & $10(76,9)$ & 0,002 \\
\hline Propósito do sítio & $2(8,3)$ & $9(69,2)$ & $<0,001$ \\
\hline Responsáveis pela seleção do conteúdo & $14(58,3)$ & $7(53,8)$ & 1,000 \\
\hline Não põe em risco a privacidade dos usuários & $22(91,7)$ & $12(92,3)$ & 1,000 \\
\hline Chat e espaço para dúvidas, reclamações e sugestões & $9(37,5)$ & $7(53,8)$ & 0,489 \\
\hline Algum efeito visual ou sonoro que desvie a atenção do entendimento das informações & $3(12,5)$ & $1(7,7)$ & 1,000 \\
\hline Voltado para alguma especialidade & $9(37,5)$ & $1(7,7)$ & 0,065 \\
\hline Responsável pela criação & $16(66,7)$ & $2(15,4)$ & 0,005 \\
\hline Responsável pela atualização & $14(58,3)$ & $2(15,4)$ & 0,017 \\
\hline Patrocinadores & $9(37,5)$ & $12(92,3)$ & 0,002 \\
\hline Responsável técnico identificado & $6(25,0)$ & $6(46,2)$ & 0,274 \\
\hline Informações ilógicas da boa compreensão terapêutica e farmacocinética & $4(16,7)$ & $3(23,1)$ & 0,678 \\
\hline Artigos submetidos a um editor de revisão & $14(58,3)$ & $8(61,5)$ & 1,000 \\
\hline
\end{tabular}


Nos critérios de procedência, foram observadas baixas frequências tanto nos sítios sobre saúde $(8,3 \%)$ como nos sítios sobre medicamentos $(7,7 \%)$, em relação às informações das universidades. Verificou-se uma frequência mais elevada de órgãos públicos nos sítios sobre saúde em relação aos sítios sobre medicamentos. Nos sítios sobre saúde, verificou-se uma frequência mais elevada de outras instituições reconhecidamente qualificadas (70,8\%) (Tabela 3).
Nos espaços públicos ou em páginas de aconselhamento nos sítios sobre saúde, eram fornecidas informações sobre produtos, serviços e atenção médica personalizada, enquanto a demanda por serviços especializados era muito baixa. Alguns sítios induziam consultas médicas e prescrições medicamentosas.

Nenhum dos critérios de procedência apresentou diferença estatisticamente significante entre os sítios investigados (Tabela 3).

Tabela 3. Critérios de procedência

\begin{tabular}{|c|c|c|c|}
\hline \multirow{2}{*}{ Variáveis } & Sítio sobre saúde $(\mathrm{n}=24)$ & Sítio sobre medicamento $(n=13)$ & \multirow{2}{*}{ Valor de $\mathrm{p}$} \\
\hline & n $(\%)$ & n (\%) & \\
\hline Fontes profissionais & $19(79,2)$ & $11(84,6)$ & 1,000 \\
\hline Entidades & $5(20,8)$ & $2(15,4)$ & 1,000 \\
\hline Universidades & $2(8,3)$ & $1(7,7)$ & 1,000 \\
\hline Órgãos públicos (municipal, estadual e federal) & $9(37,5)$ & $3(23,1)$ & 0,476 \\
\hline Órgãos privados & $5(20,8)$ & $9(69,2)$ & 1,000 \\
\hline Outras instituiç̧ões reconhecidamente qualificadas & $17(70,8)$ & $9(69,2)$ & 1,000 \\
\hline Voltado para alguma especialidade & $9(37,5)$ & $1(7,7)$ & 0,065 \\
\hline
\end{tabular}

\section{DISCUSSÃO}

Com base nos critérios adotados de acesso, aparência, organização, honestidade, transparência, responsabilidade e procedência, para avaliar as diferenças das informações geradas nos sítios sobre saúde e sobre medicamentos, somente o critério honestidade apresentou diferenças estatisticamente significantes em todos seus itens.

Por outro lado, o estudo revelou que as informações geradas nos sítios sobre saúde e sobre medicamentos se relacionam diretamente com o mercado e a comercialização de produtos farmacêuticos. Os sítios sobre medicamentos usam fontes comerciais, como indústrias farmacêuticas, distribuidoras e farmácias virtuais, para fornecer informações. O dispositivo disponível para as informações sobre medicamentos são as bulas produzidas pela indústria ou folhetos informativos. A qualidade dos textos das bulas dos medicamentos comercializados no Brasil é duvidosa, pois omitem ou abandonam informações importantes ${ }^{(21)}$.

Os resultados mostram uma situação preocupante nos sítios sobre medicamentos, não apenas por conta do aspecto legal ${ }^{(22)}$, mas, particularmente, por influência do mercado: eles apresentam nomes de patrocinadores, apontando sua influência e, ainda, mostraram-se dispostos a oferecer ajuda aos usuários. Anúncios de produtos são vistos como desfavoráveis nos Estados Unidos e indicam que a informação pode ser inclinada à comercialização pelos usuários americanos ${ }^{(23)}$.
Os usuários devem estar cientes de que as informações sobre medicamentos devem ir além do interesse de ampliar as vendas, ou seja, deve fornecer a esses usuários informações adequadas sobre medicamentos ${ }^{(24)}$.

Evidenciou-se que alguns sítios induziam consultas médicas e prescrições medicamentosas, embora seja de fundamental importância reconhecer que o aconselhamento médico seja uma atitude complementar, mas que não substitui a relação médico-paciente ${ }^{(19)}$.

A ausência da identificação da responsabilidade técnica coloca em questão a atuação dos órgãos de vigilância sanitária ${ }^{(18,22)}$, responsáveis por fiscalizar os sítios sobre saúde e sobre medicamentos. A fiscalização, por parte dos órgãos de vigilâncias sanitárias, precisa estar mais presentes nestes espaços, para se evitarem abusos. Um dos papéis desses órgãos é monitorar esses sítios, para que sejam fornecidas informações seguras e precisas, e para que sirvam de fonte de informações sobre medicamentos e sobre saúde de qualidade para a sociedade.

Essa situação pode ser muito perigosa para o usuário, por se tratar de sítios que não apresentam profissionais habilitados a fornecerem informações seguras sobre os processos saúde-doença ou medicamentos, podendo levar o usuário a usar incorretamente medicamentos ou a gerar agravos à saúde. Estudo mostrou que os usuários americanos com doença crônica têm 1,3 chances de usar mais internet para procurar informação sobre seu problema do que os que não têm doença crônica ${ }^{(23)}$. 
Critérios básicos da legislação, para garantir a qualidade da informação ${ }^{(18,22)}$, como fornecer links com mensagens de alerta, links que levam o usuário direto ao sítio da ANVISA e atualizações constantes e visíveis ao fornecedor, não são seguidos.

Os espaços para dúvidas e sugestões são insuficientes. Alguns sítios, no entanto, disponibilizam aos usuários espaços para tirar suas dúvidas sobre patologia e medicamentos que usam. Há ainda sítios que expõem discussões em fóruns, as quais podem ser vistas por qualquer outro usuário, colocando em risco a privacidade dos usuários que não desejam exposição ${ }^{(18)}$.

Os critérios da qualidade das informações na internet variam ainda quanto à origem do sítio, atualização, processo editorial de elaboração, interesses do provedor e outros ${ }^{(25)}$. Quando esses critérios são adotados, podem ajudar o profissional de saúde em sua tarefa de cuidado ao paciente. Entretanto, é de fundamental importância que essas informações não sofram influências externas, que sejam repassadas de forma clara e precisa, além de possuírem fontes confiáveis. Daí, percebem-se nitidamente os riscos inerentes à disponibilidade de informações sobre saúde e medicamentos pela internet.

\section{CONCLUSÕES}

Os achados deste estudo constatam que o critério honestidade é o elemento fundamental para garantir a qualidade das informações gerada nos sítios sobre saúde e sobre medicamentos. É preciso ainda considerar critérios como acesso, aparência, organização, transparência, responsabilidade e procedência.

A qualidade das informações geradas nos sítios sobre saúde e sobre medicamentos apresenta diferenças importantes, devendo ser exigida uma organização mais sistemática sobre os temas apresentados em seus ciberespaços e estabelecidos parâmetros de qualidade da informação, como precisão, durabilidade, atualização e autoridades de informações disseminadas na rede.

As informações sobre saúde e medicamentos devem ser fornecidas, de forma que seja necessário um comprometimento ético por parte produtor da informação frente ao usuário.

\section{REFERÊNCIAS}

1. Eysenbach $G$, Diepgen TL. Labeling and filtering of medical information on the internet. Methods Inf Med. 1999;38(2):80-8.

2. Eysenbach G, Kohler Ch. What is the prevalence of health-related searches on the World Wide Web? Qualitative and quantitative analysis of search engine queries on the Internet. AMIA Annu Symp Proc. 2003:225-9.

3. Madden M. The changing picture of who's online and what they do [Internet].
Pew Internet \& American Life Project. 2003 Dec 22. [cited 2012 Sep 8]. Available from: http://www.pewinternet.org/ /media//Files/Reports/2003/ PIP_Online_Pursuits_Final.PDF.PDF

4. Cline RJW, Haynes KM. Consumer health information seeking on the Internet: the state of the art. Health Educ Res. 2001;16(6):671-92.

5. Spink A, Yang Y, Jansen J, Nykanen P, Lorence DP, Ozmutlu S, et al. A study of medical and health queries to web search engines. Health Info Libr J. 2004; 21(1):44-51.

6. Gondim, APS, Falcão, CB. Avaliação das farmácias virtuais brasileiras. Rev Saúde Pública. 2007;41(2):297-300.

7. Silva EV, Castro, LLC. Infodemiologia: uma abordagem epidemiológica da informação. Rev Espaço Saúde. 2007;8(2):39-43.

8. Aquino DS. Por que o uso racional de medicamentos deve ser uma prioridade? Ciênc Saúde Coletiva. 2008;13(Supl):733-6.

9. Gatyas G, Savage C. IMS: Forecasts Global Pharmaceutical Market Growth of 4 - 6\% in 2010; Predicts 4 - 7\% Expansion Through 2013. [cited 2012 set 12]. Available from: http://www.imshealth.com/portal/site/ims/menuitem.d2 48e29c86589c9c30e81c033208c22a/?vgnextoid =4b8c410b6c718210Vgn VCM100000ed152ca2RCRD\&vgnextfmt $=$ default

10. Ribbink, K. Olá Brazil: Latin America's biggest market accelerates. Pharmavoice. 2011. [cited 2011 Mar 5] Available from: http://www.imshealth. com/imshealth/Global/Content/IMS\%20in\%20the\%20News/Documents/ PharmaVoice,\%20January,\%20Pharmerging-Brazil,\%20Nilton\%20Paletta.pdf

11. Parker M, Gray J. What is the role of clinical ethics support in the era of emedicine? J Med Ethics. 2001;27(Suppl 1):i33-5.

12. e-Health Ethics Initiative. e-Health Ethics Draft Code (Feb 18). J Med Internet Res. 2000;2(1):E2.

13. Rodrigues R. Ethical and legal issues in interactive health communications: a call for international cooperation. J Med Internet Res. 2000;2(1):E8.

14. Lopes IL. Estudos sobre qualidade da informação em Saúde na Web e a visão de entidades de classe brasileiras [Internet]. [citado 2012 Set 8]. Disponível em: www.tempusactas.unb.br/index.php/tempus/article/viewFile/398/381

15. Mack J, Phil MS. The internet healthcare coalition. J Med Internet Res. 2000; 2(1):E3.

16. World Health Organization. Medical Products and the Internet: A guide to finding reliable information - Regulatory Support Series No. 008 [Internet] Geneva: WHO; 1999. [cited 2012 Set 12]. Available from: http://apps.who. int/medicinedocs/en/d/Js2277e/

17. Winker MA, Flanagin A, Chi-Lum B, White J, Andrews K, Kennett RL, et al. Guidelines for medical and health information sites on the internet: principles governing AMA web sites. JAMA. 2000;283(12):1600-6.

18. Conselho Federal de Medicina (CFM). Resolução n $n^{0} 1639$ de 10 de Julho de 2002. Normas técnicas para o uso de sistemas informatizados para a guarda e manuseio do prontuário médico. Brasília (DF):CFM; 2002.

19. Conselho Regional de Medicina do Estado de São Paulo (CREMESP). Resolução nº 097 de 9 de março de 2001. Manual de princípios éticos para sites de medicina e saúde. São Paulo: CRM; 2001.

20. Sullivan D. comScore: US has most searches; China Slowest Growth; Google Tops Worldwide in 2009 [Internet]. [cited 2012 Set 8]. Available from: http:// searchengineland.com/comscore-us-most-searches-china-slowest-34217

21. Gonçalves AS, Melo G, Tokarski MHL, Branco AB. Bula de medicamentos como instrumento de informação técnico-científica. Rev Saúde Pública. 2002;36(1):33-9.

22. Brasil. Ministério da Saúde. Agência Nacional de Vigilância Sanitária. Consulta pública nº 20, de 21 de março de 2001. Brasília (DF): MS/ANVISA, mar. 2001.

23. Bansil P, Keenan NL, Zlot Al, Gilliland JC. Health-related information on the web: results from the health styles survey, 2002-2003. Prev Chronic Dis. 2006;3(2):A36

24. Barros JAC. Há riscos nas informações sobre saúde e medicamentos disseminados pela internet? Saúde em Debate. 2004;28(66):75-9.

25. Risk A, Dzenowagis J. Review of internet health information quality initiatives. J Med Internet Res. 2001;3(4):E28. 
Anexo 1. Formulário elaborado para coleta de dados dos sítios analisados, com base nos critérios da qualidade da informação na internet, estabelecidos pela Organização Mundial da Saúde, e no Código Internacional de Ética para sítios de saúde e serviços na internet

\section{FORMULÁRIO}

Dados de cadastro

Data da visita:

Nome do sítio:

Dados sobre a origem da informação da página

Nome do autor:

E-mail:

Telefone:

Profissional da área de saúde responsável:

Dados de análise das informações do sítio

A que propósito o sítio se destina?

I - Que tipo de produto ele oferece?

Espaço público

Produtos

Assessoria

Aconselhamento

Serviços

Atenção médica personalizada

Il - Apresenta nome do:

Responsável pela criação do sítio

Mantedor ou responsável pelas atualizações

Patrocinadores
Avaliador:

URL do website de operação:

URL do website de operação das informações
Cidade:

Fax:

$\begin{array}{ll}\square \text { Informativo } & \\ \text { Sim } & \text { Não } \\ \square & \square \\ \square & \square \\ \square & \square \\ \square & \square \\ \square & \square \\ \square & \square\end{array}$

Estado:
Registro no Conselho:

III - Há interferência dos autores das informações e dos patrocinadores no conteúdo e linha editorial dos sítios?

$\square$ Não interfere

IV - As informações apresentadas nos sítios na internet são de:

Fácil entendimento e linguagem objetiva

Exata e cientificamente fundamentada (profissional qualificado com base em estudos e pesquisas)

Fácil acesso

Com que frequência o sítio é atualizado?

V - Está visível a data da publicação ou revisão da informação? $\quad \square \operatorname{Sim} \quad \square$ Não

VI - Os sítios citam as fontes utilizadas nas informações? $\quad \square$ Sim $\quad \square$ Não

VII - Possui o nome e o contato com os responsáveis pela seleção dos conteúdos no sítio? $\quad \square$ Sim $\quad \square$ Não

VIII - O sítio põe em risco, de alguma maneira, a privacidade das informações dos usuários? $\quad \square$ Sim $\quad \square$ Não

IX - O sítio possui um chat ou espaço para dúvidas, reclamações e sugestões? $\quad \square$ Sim $\quad \square$ Não

$X$ - As informações fornecidas no sítio utilizam que tipo de fontes?

$\begin{array}{lll} & \text { Sim } & \text { Não } \\ \text { Fontes profissionais } & \square & \square \\ \text { Entidades } & \square & \square \\ \text { Universidade } & \square & \square \\ \text { Órgãos públicos } & \square & \square \\ \text { Órgãos privados } & \square & \square \\ \text { Instituições reconhecidamente qualificadas } & \square & \square\end{array}$

$\mathrm{XI}$ - Os responsáveis técnicos pelas informações estão identificados no sítio com nome e registro no conselho? $\quad \square$ Sim $\square$ Não

XII - Existem informações ilógicas conforme uma boa compreensão terapêutica e farmacocinética? $\quad \square$ Sim $\quad \square$ ão

XIII - Há publicidades inadequadas na página? $\quad \square \operatorname{Sim} \quad \square$ Não

XIV - Há algum efeito visual que desvia a atenção da observação e entendimento do conteúdo informado? $\quad \square \operatorname{Sim} \quad \square$ Não

$\mathrm{XV}$ - A página fornece endereços (Links) para outros sítios relevantes? $\quad \square$ Sim $\square$ Não

XVI - Os artigos parecem ter sido submetidos a um editor ou processo de revisão por pares (peer review)? $\quad \square \operatorname{Sim} \quad \square$ Não

XVII - As informações são organizadas em uma forma lógica? $\quad \square$ Sim $\quad \square$ Não

XVIII - É visível o número de usuários que já acessaram o sítio? $\quad \square$ Sim Quantos?

$\square$ Não

XIX - O conteúdo da página é voltado para alguma especialidade?

$\square \operatorname{Sim} \quad$ Qual? $\square$ Não 